\title{
ОЦЕНИВАНИЕ КОЭФФИЦИЕНТОВ ЛИНЕИНОЙ РЕГРЕССИИ КАК АНТАГОНИСТИЧЕСКАЯ ИГРА
}

\author{
(Представил Н. Алумяэ)
}

Рассмотрим регрессионную модель

$$
\mathbf{Y}=\mathbf{X} \theta+\varepsilon,
$$

где $\mathbf{Y}-n$-мерный наблюдаемый случайный вектор, $\mathcal{E}-n$-мерный ненаблюдаемый случайный вектор, причем $E(\varepsilon \mid \theta)=0$ и $E\left(\varepsilon^{\text {क }}{ }^{\mathrm{T}} \mid \theta\right)=$ $=\sigma^{2} I_{n}, I_{n}-$ единичная матрица порядка $n$, элементы матрицы $\mathbf{X}$ порядка $n \times m$ и $\sigma^{2}-$ известные коэффициенты, а $\theta-m$-мерный вектор, подлежащий оцениванию.

Пусть априори известно, что оцениваемый вектор $\theta$ принадлежит $m$-мерному эллипсоиду $L(\mathbf{A})=\left\{\theta: \theta^{\mathrm{T}} \mathbf{A} \theta \leqslant 1\right\}$, где $\mathbf{A}-$ известная неотрицательно определенная матрица порядка $m$. Оценивание вектора $\theta$ с помощью статистики $\hat{\theta}(\mathbf{Y})$, с одной стороны, и выбор вектора $\theta$, с другой стороны, можно рассматривать как игру двух лиц $\left[{ }^{1}\right]$ : статистики $\hat{\theta}(\mathbf{Y})$ образуют класс стратегий первого игрока, а векторы $\theta$, принадлежащие эллипсоиду $L(\mathbf{A})$, - класс стратегий второго игрока. Ограничим стратегии первого игрока линейными по наблюдениям статистиками, т: е.

$$
\hat{\theta}(\mathbf{Y})=\mathbf{T Y},
$$

где $\mathbf{T}$ - произвольная матрица порядка $m \times n$, составленная из неслучайных элементов. За функцию игры примем квадратичный риск

$$
r(\theta, \mathbf{T})=E\left\{(\theta-\mathbf{T Y})^{\mathrm{T}} \mathbf{P}(\theta-\mathbf{T Y}) / \theta\right\},
$$

где $\mathbf{P}$ - заданная неотрицательно определенная матрица порядка $m$ и ранга $R(\mathbf{P})=k \leqslant m$. Таким образом, при использовании первым игроком матрицы T, а вторым - вектора $\theta$ величина $r(\theta, \mathbf{T})$ есть проигрыш первого игрока и выигрыш второго.

B $\left[{ }^{2-4}\right]$ рассматривалась минимаксная задача, т. е. поиск матрицы $\mathbf{T}_{0}$ такой, что

$$
\inf _{\mathbf{T}} \sup _{\theta \in L(\mathbf{A})} r(\theta, \mathbf{T})=\sup _{\theta \in L(\mathbf{A})} r\left(\theta, \mathbf{T}_{0}\right) .
$$

В настоящей работе решается задача максимизации гарантированного выигрыша второго игрока, т. е.

$$
\inf _{\mathbf{T}} r(\theta, \mathbf{T}) \rightarrow \sup _{\theta \in L(A)}
$$

Кроме того, выводится условие, при котором игра является вполне определенной и которое позволяет решать минимаксную задачу более простым методом, чем это сделано в $\left[{ }^{2,3}\right]$.

Пусть $\mathrm{p}_{1}, \ldots, \mathrm{p}_{k}$ - ортонормированные собственные векторы мат- 
рицы $\mathbf{P}$, соответствующие ненулевым собственным числам $\lambda_{1}, \ldots, \lambda_{k}$. Тогда $\mathbf{P}=\sum_{i=1}^{k} \lambda_{i} \mathbf{p}_{i} \mathbf{p}_{i}^{\mathrm{T}}$, а так как $\left[{ }^{2}\right]$

$$
r(\theta, \mathbf{T})=\sigma^{2} \operatorname{tr} \mathbf{T}^{\mathrm{T}} \mathbf{P} \mathbf{T}+\theta^{\mathrm{T}}\left(\mathbf{I}_{m}-\mathbf{T X}\right)^{\mathrm{T}} \mathbf{P}\left(\mathbf{I}_{m}-\mathbf{T X}\right) \theta,
$$

To

$$
r(\theta, \mathbf{T})=\sigma^{2} \sum_{i=1}^{k} \lambda_{i} \mathbf{p}_{i}^{\mathrm{T}} \mathbf{T T}^{\mathrm{T}} \mathbf{p}_{i}+\sum_{i=1}^{h} \lambda_{i}\left(\mathbf{p}_{i}^{\mathrm{T}}\left(\mathbf{I}_{m}-\mathbf{T X}\right) \theta\right)^{2}
$$

Дифференцируя по векторам $\mathrm{T}^{\mathrm{T}} \mathrm{p}_{i}, i=1,2, \ldots, k$, выпуклую по ним функцию $r(\theta, \mathbf{T})$, получаем [5]

$$
\frac{\partial r(\theta, \mathbf{T})}{\partial \boldsymbol{T}^{\mathrm{T}} \boldsymbol{p}_{i}}=2 \sigma^{2} \lambda_{i} \mathbf{T}^{\mathrm{T}} \mathbf{p}_{i}+2 \lambda_{i} \mathbf{p}_{i}^{\mathrm{T}}\left(\mathbf{I}_{m}-\mathbf{T} \mathbf{X}\right) \theta(-\mathbf{X} \theta)
$$

и, следовательно, векторы, на которых достигается минимальное значение функции $r(\theta, \mathbf{T})$, представимы в виде

$$
\mathbf{T}^{\mathrm{T}} \mathbf{p}_{i}=\left(\sigma^{2} \mathbf{I}_{n}+\mathbf{X} \theta \theta^{\mathrm{T}} \mathbf{X}^{\mathrm{T}}\right)^{-1} \mathbf{X} \theta \theta^{\mathrm{T}} \mathbf{p}_{i}
$$

Так как $\left[{ }^{5}\right]$

$$
\left(\sigma^{2} \mathbf{I}_{n}+\mathbf{X} \theta \theta^{\mathrm{T}} \mathbf{X}^{\mathrm{T}}\right)^{-1}=1 / \sigma^{2} \mathbf{I}_{n}-1 / \sigma^{2} \mathbf{X} \theta \theta^{\mathrm{T}} \mathbf{X}^{\mathrm{T}} /\left(\sigma^{2}+\theta^{\mathrm{T}} \mathbf{X}^{\mathrm{T}} \mathbf{X} \theta\right),
$$

To

$$
\mathbf{T}^{\mathrm{T}} \mathbf{p}_{i}=\mathbf{X} \theta \theta^{\mathrm{T}} \mathbf{p}_{i} /\left(\sigma^{2}+\theta^{\mathrm{T}} \mathbf{X}^{\mathrm{T}} \mathbf{X} \theta\right)
$$

Подставляя (4) в (3), получаем равенство

$$
\begin{gathered}
\inf _{\mathbf{T}} r(\theta, \mathbf{T})=\sigma^{2} \cdot \theta^{\mathrm{T}} \mathbf{X}^{\mathrm{T}} \mathbf{X} \theta \theta^{\mathrm{T}} \mathbf{P} \theta /\left(\sigma^{2}+\theta^{\mathrm{T}} \mathbf{X}^{\mathrm{T}} \mathbf{X} \theta\right)^{2}+ \\
+\theta^{\mathrm{T}}\left(\mathbf{I}_{m}-\mathbf{X}^{\mathrm{T}} \mathbf{X} \theta \theta^{\mathrm{T}} /\left(\sigma^{2}+\theta^{\mathrm{T}} \mathbf{X}^{\mathrm{T}} \mathbf{X} \theta\right)\right) \mathbf{P}\left(\mathbf{I}_{m}-\theta \theta^{\mathrm{T}} \mathbf{X}^{\mathrm{T}} \mathbf{X} /\left(\sigma^{2}+\theta^{\mathrm{T}} \mathbf{X}^{\mathrm{T}} \mathbf{X} \theta\right)\right) \theta,
\end{gathered}
$$

которое легко преобразуется к равенству

$$
\inf _{\mathbf{T}} r(\theta, \mathbf{T})=\sigma^{2} \theta^{\mathrm{T}} \mathbf{P} \theta /\left(\sigma^{2}+\theta^{\mathrm{T}} \mathbf{X}^{\mathrm{T}} \mathbf{X} \theta\right) .
$$

Таким образом, решение задачи сводится к поиску

$$
\sup _{\theta \in L(\mathbf{A})} \sigma^{2} \theta^{\mathrm{T}} \mathbf{P} \theta /\left(\sigma^{2}+\theta^{\mathrm{T}} \mathbf{X}^{\mathrm{T}} \mathbf{X} \theta\right) \text {. }
$$

Т еорем а 1. Для того чтобы выражение (6) было конечно, необходимо и достаточно включения

$$
\text { Ker } \mathbf{A} \cap \operatorname{Ker} \mathbf{X}^{\mathbf{T}} \mathbf{X} \subseteq \operatorname{Ker} \mathbf{P} \text {, }
$$

где, как обычно, для произвольной матриць В порядка $m \times m$ ядро $\operatorname{Ker} \mathbf{B}=\left\{\theta \in R^{m}: \mathbf{B} \theta=0\right\}$.

Доказательство. Необходимость легко доказывается от противного. Пусть условие (7) не выполнено, т. е. существует вектор $\theta_{n} \in R^{m}$ такой, что $\mathbf{A} \theta_{0}=\mathbf{X}^{\mathrm{T}} \mathbf{X} \theta_{0}=0$ и $\mathbf{P} \theta_{0} \neq 0$. Выберем последовательность векторов $\theta_{n}=n \theta_{0}, n=1,2, \ldots$. Тогда, очевидно, $\theta_{n}{ }^{\mathrm{T}} A \theta_{n}<1$ и

$$
\theta_{n}^{\mathrm{T}} \mathbf{P} \theta_{n} /\left(\sigma^{2}+\theta_{n}^{\mathrm{T}} \mathbf{X}^{\mathrm{T}} \mathbf{X} \theta_{n}\right)=1 / \sigma^{2} \cdot n^{2} \theta_{0}^{\mathrm{T}} \mathbf{P} \theta_{0} \underset{n \rightarrow \infty}{\longrightarrow} \infty \text {. }
$$

Докажем достаточность. Заметим, что если В - ненулевая неотрицательно определенная матрица, то по теореме Куранта-Фишера $\left[{ }^{6}\right]$

$$
\inf _{\theta \in \operatorname{Ker}^{\perp} \mathbf{B} \subset R^{m}} \theta^{\mathrm{T}} \mathbf{B} \theta / \theta^{\mathrm{T}} \theta=b_{1},
$$


где $b_{1}$ - минимальное положительное собственное число матрицы $\mathbf{B}$, a $\operatorname{Ker}^{\perp} \mathbf{B}$ - ортогональное дополнение ядра Ker B. Таким образом,

$$
\begin{gathered}
\sup _{\theta \in L(\mathbf{A})} \theta^{\mathrm{T}} \mathbf{P} \theta /\left(\sigma^{2}+\theta^{\mathrm{T}} \mathbf{X}^{\mathrm{T}} \mathbf{X} \theta\right) \leqslant \sup _{\theta \in L(\mathbf{A})} \theta^{\mathrm{T}} \mathbf{P} \theta / \theta^{\mathrm{T}}\left(\sigma^{2} \mathbf{A}+\mathbf{X}^{\mathrm{T}} \mathbf{X}\right) \theta \leqslant \\
\leqslant \sup _{\theta \in R^{m}} \theta^{\mathrm{T}} \mathbf{P} \theta / \theta^{\mathrm{T}}\left(\sigma^{2} \mathbf{A}+\mathbf{X}^{\mathrm{T}} \mathbf{X}\right) \theta= \\
=\sup _{\theta_{1}, \theta_{2} \in R^{m}}\left(\theta_{1}+\theta_{2}\right)^{\mathrm{T}} \mathbf{P}\left(\theta_{1}+\theta_{2}\right) /\left(\theta_{1}+\theta_{2}\right)^{\mathrm{T}}\left(\sigma^{2} \mathbf{A}+\mathbf{X}^{\mathrm{T}} \mathbf{X}\right)\left(\theta_{1}+\theta_{2}\right),
\end{gathered}
$$

где $\theta_{1} \in \operatorname{Ker}\left(\sigma^{2} \mathbf{A}+\mathbf{X}^{\mathrm{T}} \mathbf{X}\right), \theta_{2} \in \operatorname{Ker}^{\perp}\left(\sigma^{2} \mathbf{A}+\mathbf{X}^{\mathrm{T}} \mathbf{X}\right)$. В силу условия (7) теоремы и равенства (8) получаем

$$
\begin{gathered}
\sup _{\theta \in L(\mathbf{A})} \theta^{\mathrm{T}} \mathbf{P} \theta /\left(\sigma^{2}+\theta^{\mathrm{T}} \mathbf{X}^{\mathrm{T}} \mathbf{X} \theta\right) \leqslant \sup _{\theta_{2} \in \operatorname{Ker}^{\perp}\left(\sigma^{2} \mathbf{A}+\mathbf{x}^{\mathrm{T}} \mathbf{X}\right)} \theta_{2}^{\mathrm{T}} \mathbf{P} \theta_{2} / \theta_{2}^{\mathrm{T}}\left(\sigma^{2} \mathbf{A}+\mathbf{X}^{\mathrm{T}} \mathbf{X}\right) \theta_{2} \leqslant \\
\leqslant\left[\sup _{\theta \in R^{m}} \theta^{\mathrm{T}} \mathbf{P} \theta / \theta^{\mathrm{T}} \theta\right] /\left[\inf _{\theta \in \operatorname{Ker}^{\perp}\left(\sigma^{2} \mathbf{A}+\mathbf{X}^{\mathrm{T}} \mathbf{X}\right)} \theta^{\mathrm{T}}\left(\sigma^{2} \mathbf{A}+\mathbf{X}^{\mathrm{T}} \mathbf{X}\right) \theta / \theta^{\mathrm{T}} \theta\right]<\infty,
\end{gathered}
$$

что полностью доказывает теорему.

Те орем а 2. Пусть выполнено условие теоремы 1. Тогда

$$
\sup _{\theta \in L(A)} \inf _{\mathbf{T}} r(\theta, \mathbf{T})=\sigma^{2} f_{1}
$$

где $f_{1}$ - максимальное из чисел, удовлетворяющих равенству

$$
\mathbf{P} \theta=f\left(\sigma^{2} \mathbf{A}+\mathbf{X}^{\mathrm{T}} \mathbf{X}\right) \theta
$$

для какого-либо $\theta \in \operatorname{Ker}^{\perp}\left(\sigma^{2} \mathbf{A}+\mathbf{X}^{\mathrm{T}} \mathbf{X}\right)$.

Доказ ательство. Очевидно, что

$$
\begin{gathered}
\sup _{\theta \in L(\mathbf{A})} \theta^{\mathrm{T}} \mathbf{P} \theta /\left(\sigma^{2}+\theta^{\mathrm{T}} \mathbf{X}^{\mathrm{T}} \mathbf{X} \theta\right) \leqslant \sup _{\theta \in L(\mathbf{A})} \frac{\theta^{\mathrm{T}} \mathbf{P} \theta}{\theta^{\mathrm{T}}\left(\sigma^{2} \mathbf{A}+\mathbf{X}^{\mathrm{T}} \mathbf{X}\right) \theta} \leqslant \\
\quad \leqslant \sup _{\theta \in R^{m}} \frac{\theta^{\mathrm{T}} \mathbf{P} \theta}{\theta^{\mathrm{T}}\left(\sigma^{2} \mathbf{A}+\mathbf{X}^{\mathrm{T}} \mathbf{X}\right) \theta}=f_{1},
\end{gathered}
$$

причем последнее равенство реализуется на векторе $\theta_{1}$, удовлетворяющем равенству

$$
\mathbf{P} \theta_{1}=f_{1}\left(\sigma^{2} \mathbf{A}+\mathbf{X}^{\mathrm{T}} \mathbf{X}\right) \theta_{1}, \quad \theta_{1} \in \operatorname{Ker}^{\perp}\left(\sigma^{2} \mathbf{A}+\mathbf{X}^{\mathrm{T}} \mathbf{X}\right) .
$$

Пусть $\mathbf{A} \theta_{1} \neq 0$, тогда можно считать, что $\theta_{1}{ }^{\mathrm{T}} \mathbf{A} \theta_{1}=1$ и, таким образом, в (9) везде получаем равенства. Пусть теперь $\mathbf{A} \theta_{1}=0$. Выберем $\theta_{n}=n \theta_{1}$ и получим

$$
\sigma^{2} \theta_{n}^{\mathrm{T}} \mathbf{P} \theta_{n} /\left(\sigma^{2}+\theta_{n}^{\mathrm{T}} \mathbf{X}^{\mathrm{T}} \mathbf{X} \theta_{n}\right) \underset{n \rightarrow \infty}{\longrightarrow} \theta_{1}^{\mathrm{T}} \mathbf{P} \theta_{1} / \theta_{1}^{\mathrm{T}} \mathbf{X}^{\mathrm{T}} \mathbf{X} \theta_{1}=f_{1}
$$

Таким образом, в обоих случаях неравенства (9) в действительности являются равенствами, что и доказывает теорему.

3 а меч а н и е 1 . Нетрудно показать, что число $f_{1}$, определенное в теореме, является максимальным собственным числом матрицы $\left(\sigma^{2} \mathbf{A}+\mathbf{X}^{\mathrm{T}} \mathbf{X}\right)^{+1 / 2} \mathbf{P}\left(\sigma^{2} \mathbf{A}+\mathbf{X}^{\mathrm{T}} \mathbf{X}\right)^{+1 / 2}$, где $(\cdot)^{+}$- обратная матрица Мура $\left[{ }^{5}\right]$.

Определим теперь условия, при которых описанная игра является вполне определенной, т. е.

$$
\inf _{\mathbf{T}} \sup _{\theta \in L(\mathbf{A})} r(\theta, \mathbf{T})=\sup _{\theta \in L(\mathbf{A})} \inf _{\mathbf{T}} r(\theta, \mathbf{T}) .
$$

В частности, в $\left[{ }^{2}\right]$ показано, что при $R(\mathbf{P})=1$ равенство (10) имеет место для любых матриц $\mathbf{A}$ и $\mathbf{X}^{\mathbf{T}} \mathbf{X}$. Рассмотрим случай, когда $R(\mathbf{P}) \geqslant 2$. 
Для полной определенности игры необходимо и достаточно существования седловой точки $\left[{ }^{1}\right]$, т. е. таких стратегий $\mathbf{T}_{0}$ и $\theta_{0} \in L(\mathbf{A})$, что

$$
r\left(\theta, \mathbf{T}_{0}\right) \leqslant r\left(\theta_{0}, \mathbf{T}_{0}\right) \leqslant r\left(\theta_{0}, \mathbf{T}\right) \forall \mathbf{T}, \theta \in L(\mathbf{A}) .
$$

Из правого неравенства (11) получаем, что $\mathbf{T}_{0}=\theta_{0} \theta_{0}{ }^{\mathrm{T}} \mathbf{X}^{\mathrm{T}} /\left(\sigma^{2}+\theta_{0}^{\mathrm{T}} \mathbf{X}^{\mathrm{T}} \mathbf{X} \theta_{0}\right)$, а из левого неравенства $-\theta_{0}=\theta_{1}$, где вектор $\theta_{1}$ из теоремы 2. Таким образом, если игра определена, то стратегии $\mathbf{T}_{1}=\theta_{1} \theta_{1}{ }^{\mathrm{T}} \mathbf{X}^{\mathrm{T}} /\left(\sigma^{2}+\theta_{1}{ }^{\mathrm{T}} \mathbf{X}^{\mathrm{T}} \mathbf{X} \theta_{1}\right)$ и $\theta_{1}$ образуют седловую точку. Используя равенство $\mathbf{P} \theta_{1}=f_{1}\left(\sigma^{2} \mathbf{A}+\right.$ $\left.+\mathbf{X}^{\mathrm{T}} \mathbf{X}\right) \theta_{1}$, получаем

$$
\begin{gathered}
r\left(\theta, \mathbf{T}_{1}\right)=\sigma^{2} \theta_{1}^{\mathrm{T}} \mathbf{X}^{\mathrm{T}} \mathbf{X} \theta_{1} \theta_{1}^{\mathrm{T}} \mathbf{P} \theta_{1} /\left(\sigma^{2}+\theta_{1}^{\mathrm{T}} \mathbf{X}^{\mathrm{T}} \mathbf{X} \theta_{1}\right)^{2}+\theta^{\mathrm{T}}\left[\mathbf{P}-2 \mathbf{X}^{\mathrm{T}} \mathbf{X} \theta_{1} \theta_{1}^{\mathrm{T}} \mathbf{P} \times\right. \\
\left.\times\left(\sigma^{2}+\theta_{1}^{\mathrm{T}} \mathbf{X}^{\mathrm{T}} \mathbf{X} \theta_{1}\right)^{-1}+\theta_{1}^{\mathrm{T}} \mathbf{P} \theta_{1} \cdot \mathbf{X}^{\mathrm{T}} \mathbf{X} \theta_{1} \theta_{1}^{\mathrm{T}} \mathbf{X}^{\mathrm{T}} \mathbf{X} /\left(\sigma^{2}+\theta_{1}^{\mathrm{T}} \mathbf{X}^{\mathrm{T}} \mathbf{X} \theta_{1}\right)^{2}\right] \theta= \\
=\sigma^{2} \cdot f_{1}^{2} \theta_{1}^{\mathrm{T}} \mathbf{X}^{\mathrm{T}} \mathbf{X} \theta_{1} / \theta_{1}^{\mathrm{T}} \mathbf{P} \theta_{1}+\theta^{\mathrm{T}} \mathbf{B} \theta,
\end{gathered}
$$

где $\mathbf{B}=\mathbf{P}+\left(f_{1}^{2} \sigma^{4} \mathbf{A} \theta_{1} \theta_{1}^{\mathrm{T}} \mathbf{A}-\mathbf{P} \theta_{1} \theta_{1}^{\mathrm{T}} \mathbf{P}\right) / \theta_{1}^{\mathrm{T}} \mathbf{P} \theta_{1}$. Отсюда следует

Те орем а 3. Для полной определенности игры необходимо и достаточно, чтобы

$$
\sup _{\theta \in L(\mathbf{A})} \theta^{\mathrm{T}} \mathbf{B} \theta=\theta_{1}^{\mathrm{T}} \mathbf{B} \theta_{1} .
$$

З а меч ание 2. Условие (12) эквивалентно тому, что вектор $\mathbf{A}^{1 / 2} \theta_{1}$ является собственным вектором матрицы $\mathbf{A}^{+1 / 2} \mathbf{B} \mathbf{A}^{+1 / 2}$, соответствующим максимальному собственному числу этой матрицы.

3 а меч ание 3 . Условие $A \theta_{1} \neq 0$ необходимо для полной определенности игры, так как в противном случае $\theta_{1}{ }^{\mathrm{T}} \mathbf{B} \theta_{1}=0$, что противоречит (12).

3 а м е чание 4. Условие $\mathrm{Ker} \mathbf{A} \subseteq \operatorname{Ker} \mathbf{P}$ необходимо для полной определенности игры, так как в противном случае $\sup _{\theta \in L(\mathbf{A})} \theta^{\mathrm{T}} \mathbf{B} \theta=\infty$, что противоречит (12)

Более простым выглядит решение задачи об определенности игры при условии, сформулированном в $\left[{ }^{3}\right]$, т. е. при существовании невырожденной матрицы $\mathrm{C}$ такой, что матрицы $\overline{\mathbf{A}}=\mathrm{C}^{\mathrm{T}} \mathrm{AC}, \overline{\mathrm{P}}=\mathrm{C}^{\mathrm{T}} \mathbf{P C}, \overline{\mathbf{X}^{\mathrm{T}} \mathbf{X}}=$ $=\mathbf{C}^{\mathrm{T}} \mathbf{X}^{\mathrm{T}} \mathbf{X C}$ являются диагональными с диагональными элементами $a_{1}^{2}, \ldots, a_{m}^{2}, p_{1}^{2}, \ldots, p_{m}^{2}, x_{1}^{2}, \ldots, x_{m}^{2}$ соответственно. Очевидно, что задача с матрицами $\mathbf{A} ; \mathbf{P}$ и $\mathbf{X}^{\mathrm{T}} \mathbf{X}$ эквивалентна задаче с матрицами $\overline{\mathbf{A}}, \overline{\mathbf{P}}$ и $\overline{\mathbf{X}^{\mathrm{T}} \mathbf{X}}$. Пусть для определенности

$$
\max _{1 \leqslant i \leqslant m} \mathbf{p}_{i}^{2} /\left(\sigma^{2} a_{i}^{2}+x_{i}^{2}\right)=\mathbf{p}_{1}^{2} /\left(\sigma^{2} a_{1}^{2}+x_{1}^{2}\right)=f_{1} .
$$

Тогда у вектора $\theta_{1}$ первая компонента $1 / a_{1}$ (см. замечание 3 ), остальные компоненты - нули, а сам вектор $\theta_{1}$ удовлетворяет уравнению

$$
\overline{\mathbf{p}} \theta_{1}=f_{1}\left(\sigma^{2} \overline{\mathbf{A}}+\overline{\mathbf{X}^{\mathrm{T}} \mathbf{X}}\right) \theta_{1} \text {. }
$$

Очевидно, что справедливость условия (12) надо проверять лишь на векторах $\theta \bar{\in} \operatorname{Ker} \overline{\mathbf{A}}$ (см. замечание 4), удовлетворяющих условиям

$$
\overleftarrow{\mathbf{B}} \theta=\bar{f} \overline{\mathbf{A}} \theta, \quad \theta^{\mathrm{T}} \overline{\mathbf{A}} \theta=1
$$

при некотором $f \in R^{1}$. Число таких векторов совпадает с числом ненулевых диагональных элементов матрицы $\overline{\mathrm{A}}$, и векторы имеют вид: $1 / a_{j}\left(a_{j} \neq 0, m \geqslant j \geqslant 1\right)$ на $j$-м месте, а на остальных местах - нули. Тогда условие (12) эквивалентно тому, что при $a_{j} \neq 0$

$$
f_{1} \sigma^{2} \geqslant\left|p_{1} p_{j} /\left(a_{1} a_{j}\right)\right| \text {. }
$$


1. Wald, A. Statistical Decision Functions. Wiley, New York, 1950.

2. Кукс Я., Ольман В. Изв. АН ЭССР, Физ. Матем., 21, № 1, 66-72 (1972).

3. Кукс Я., Ольман В. Изв. АН ЭССР, Физ. Матем., 20, № 4, 480-482 (1971).

4. Rao, C. R. Ann. Statist., 4, № 6, 1023-1037, (1976).

5. Рaо С. P. Линейные статистические методы и их применения. М., «Наука», 1968.

6. Беллман Р. Введение в теорию матриц. М., «Наука», 1969.

\section{Ннститут кибернетики}

Академии наук Эстонской ССР
Поступила в редакцию 12 октября 1982

\section{OLMAN}

\section{LINEAARSE REGRESSIOONI KORDAJATE HINDAMINE KUI ANTAGONISTLIK MÄNG}

Lineaarse regressioonimudeli kordajate hindamise ülesanne on formuleeritud mänguteooria terminites. On antud sadulpunkti olemasolu tingimused, mis võimaldab mõnel juhul analüütiliselt leida esialgse minimaksülesande lahendi.

\section{OLMAN}

\section{ESTIMATION OF LINEAR REGRESSION COEFFICIENTS AS AN ANTAGONISTIC GAME}

Linear estimation of a parameter $\Theta \in R^{m}$ of a linear regression model $E \mathrm{Y}=\mathrm{X} \Theta$ is considered as an antagonistic game with the utility-loss function $r(\Theta, \mathbf{T})=$ $=E\left\{(\Theta-\mathbf{T Y})^{\mathrm{T}} \mathbf{P}(\Theta-\mathbf{T Y}) / \Theta\right\}$. The maxmin strategy in explicit form in case if an unknown parameter $\Theta$ belongs to the fixed ellipsoid $\left\{\Theta \in R^{m}: \Theta^{\mathrm{T}} \mathrm{A} \Theta \leqslant 1\right\}$ is given. Conditions for the existence of a saddle point are obtained that provide the solution of the minmax estimation problem. The solution of minmax estimation problem in case if $\mathbf{X}^{\mathrm{T}} \mathbf{X}, \mathbf{A}$ and $\mathbf{P}$ have the same eigenvectors, is given as an example. 